\title{
Prädiktion von maschineller Wahrnehmungsleistung beim automatisierten Fahren
}

\author{
Klaus Dietmayer
}

\section{Inhaltsverzeichnis}

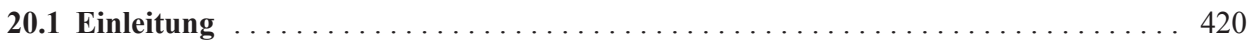

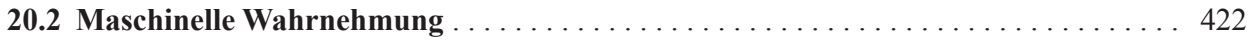

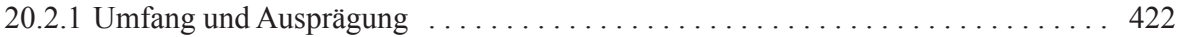

20.2.2 Ausprägung von Umgebungsmodellen . . . . . . . . . . . . . . . . . . 424

20.3 Methoden zum Umgang mit Unsicherheiten der maschinellen Wahrnehmung . . . . 425

20.3.1 Unsicherheitsdomänen ... . . . . . . . . . . . . . . . . . . . . . 425

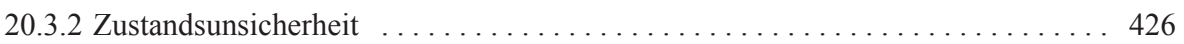

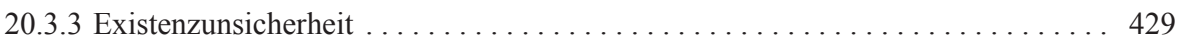

20.3.4 Klassenunsicherheit ............................. 431

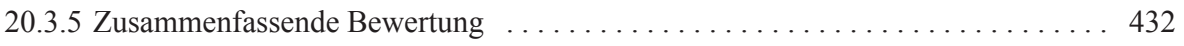

20.4 Folgerungen für die maschinelle Wahrnehmungsleistungsprädiktion $\ldots \ldots \ldots \ldots 433$

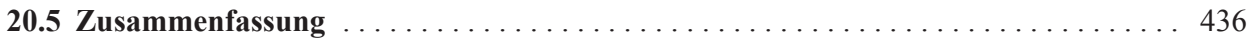

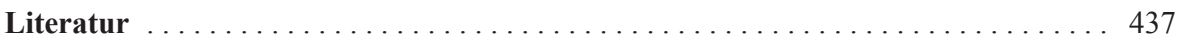

K. Dietmayer $(\square)$

Universität Ulm, Institut für Mess-, Regel- und Mikrotechnik, Deutschland klaus.dietmayer@uni-ulm.de 


\subsection{Einleitung}

Beim hoch- und vollautomatisierten Fahren ist es aufgrund der dem Fahrer zugebilligten Nebenaufgaben notwendig, dass das Fahrzeug Einschränkungen seiner maschinellen Wahrnehmung sowie Funktionseinschränkungen darauf aufbauender Verarbeitungsmodule selbst erkennt und darauf adäquat reagiert. Während man aufgrund von Simulatorstudien beim hochautomatisierten Fahren von realistischen Übergabezeiten an den Fahrer von fünf bis zehn Sekunden ausgeht [1], [2], bevor dieser die Fahraufgabe wieder sicher übernehmen kann, wäre beim vollautomatisierten Fahren der Mensch überhaupt keine Rückfallebene mehr. Das Fahrzeug müsste bei Funktionseinschränkungen vollständig selbst einen eigensicheren Zustand erreichen können. Aber auch mögliche Übergabezeiten von fünf Sekunden und mehr erfordern eine weitgehende, wenn auch zeitbegrenzte Autonomie des Fahrzeugs, um diesen Zeitraum unter allen Umständen sicher überbrücken zu können.

Um diesen Grad der Autonomie erreichen zu können, muss das Fahrzeug seine Umgebung wahrnehmen, geeignet interpretieren und daraus kontinuierlich sichere Handlungen ableiten und ausführen. Technisch wird diese Aufgabe durch einzelne, aufeinander aufbauende Verarbeitungsmodule gelöst. Eine vereinfachte Darstellung der Zusammenhänge zeigt Abb. 20.1.

Die maschinelle Wahrnehmung der Fahrumgebung erfolgt durch verschiedene am Fahrzeug verbaute Sensoren wie Kameras oder Radarsensoren. Hinzu kommen in der Regel weitere Informationen über das statische Fahrumfeld aus hochgenauen digitalen Karten. Sie sind allerdings nur dann nutzbar, wenn das Fahrzeug seine genaue Position kennt. Somit ist als weiteres Funktionsmodul auch eine Eigenlokalisation des Fahrzeugs für den Kartenabgleich erforderlich. Das Ergebnis der maschinellen Wahrnehmung ist ein dynamisches Fahrzeugumfeldmodell, in dem das eigene Fahrzeug sowie alle anderen Verkehrsteilnehmer durch individuelle Bewegungsmodelle repräsentiert sind. Ferner sollten alle relevanten

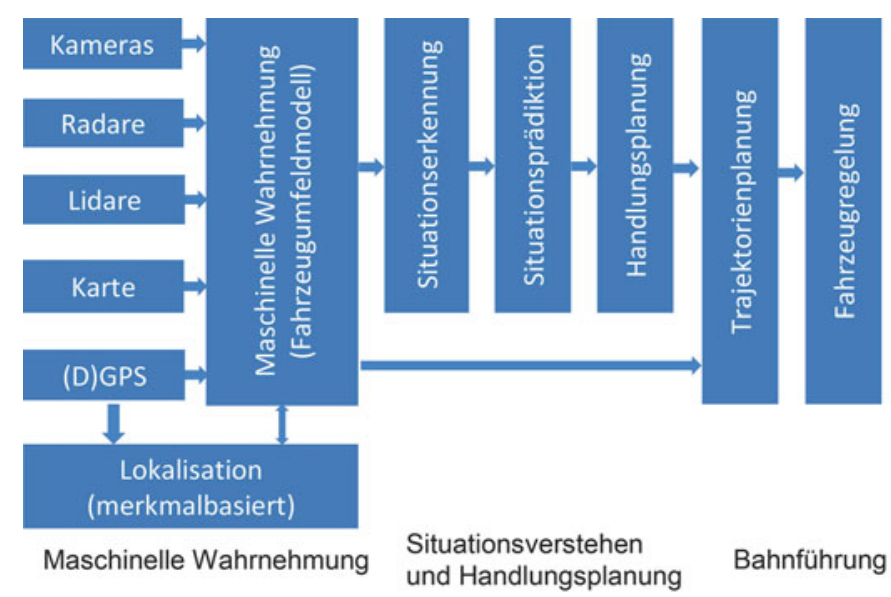

Abb. 20.1 Grobstruktur der Informationsverarbeitung bei der automatischen Fahrzeugführung 
Infrastruktureinrichtungen wie Verkehrsschilder und Lichtsignalanlagen, aber auch strukturierende Elemente wie Verkehrsinseln und Bordsteine sowie Fahrbahnmarkierungen für die Fahrstreifeneinteilung, gesperrte Flächen oder Fußgängerüberwege in Form von Zebrastreifen enthalten sein.

Aufbauend auf diesem Fahrzeugumfeldmodell werden dann bei der Situationserkennung alle Einzelkomponenten in Beziehung zueinander gesetzt, um aus den Abhängigkeiten der Einzelelemente ein maschinelles Szenenverständnis zu errechnen. Im darauf aufbauenden Modul der Situationsprädiktion werden verschiedene mögliche zeitliche Entwicklungen der Szene, auch Episoden genannt, vorausberechnet und hinsichtlich der Wahrscheinlichkeit des Eintretens bewertet. Eine Episode kennzeichnet damit in diesem Beitrag eine mögliche spezifische zeitliche Entwicklung einer erkannten Verkehrsszene, wobei der Zeithorizont im Bereich von wenigen Sekunden liegt. Auf Basis dieser situativen Informationen ermittelt das darauf aufbauende Modul eine übergeordnete Handlungsplanung. Sie könnte beispielsweise das Umfahren eines Hindernisses oder das Überholen eines langsameren Fahrzeugs vorsehen. Zur Ausführung der Pläne werden dann mögliche Trajektorien des Fahrzeugs mit einem typischen Zeithorizont von drei bis fünf Sekunden berechnet und hinsichtlich Sicherheit und Komfort bewertet. Die nach vorgebbaren Kriterien optimale Trajektorie wird von der Fahrzeugregelung ausgeführt. Der beschriebene Bearbeitungsprozess wird fortlaufend, in der Regel schritthaltend mit der sensorischen Erfassung erneut durchgeführt, um auf Aktionen und Reaktionen anderer Verkehrsteilnehmer reagieren zu können.

Die Beschreibung dieser technischen Prozesskette macht deutlich, dass ein Ausfall der maschinellen Wahrnehmung umgehend zu so großen Unsicherheiten in der Situationsbewertung führen würde, dass eine sichere Handlungsplanung und Handlungsausführung nicht mehr gewährleistet werden kann. Die Degradation des maschinellen Szenenverständnisses und der darauf aufbauenden Handlungsplanung und Handlungsausführung ist situationsabhängig; eine zuverlässige Prädiktion wird typisch aber zwei bis drei Sekunden nicht übersteigen können. Es ist somit evident, dass eine Mindestwahrnehmungsleistung schon beim hochautomatisierten Fahren aufgrund der deutlich größeren Übergabezeiträume an den Fahrer notwendig ist. Ein Komplettausfall der maschinellen Wahrnehmung ist unter allen Umständen zu vermeiden, wobei dies natürlich auch für die darauf aufbauenden Module und die Fahrzeugregelung mit ihren Sensoren und Aktoren gilt, die aber nicht im Fokus dieses Beitrags stehen.

Es stellt sich daher die Frage, ob Einschränkungen in der Funktion der maschinellen Wahrnehmung erkennbar oder sogar prädizierbar sind und, falls ja, über welchen Zeitraum. In den folgenden Abschnitten werden hierzu nach dem Stand der Technik bekannte methodische Ansätze diskutiert und darauf aufbauend mögliche Forschungsfragen abgeleitet. 


\subsection{Maschinelle Wahrnehmung}

\subsubsection{Umfang und Ausprägung}

Die maschinelle Wahrnehmung hat, wie im vorherigen Abschnitt beschrieben, die Aufgabe, alle für die Funktion des automatisierten Fahrens relevanten anderen Verkehrsteilnehmer sicher zu erkennen und der Verkehrsinfrastruktur korrekt zuzuordnen. Dies ist insbesondere deshalb notwendig, da beispielsweise für einen Fußgänger am Straßenrand ein anderes Gefährdungspotenzial besteht, als wenn er einen parallel verlaufenden, abgetrennten Fußgängerweg benutzt.

Zur maschinellen Wahrnehmung werden im Fahrzeugumfeld Sensoren verwendet, die auf Kamera- sowie Radar- und/oder Lidartechnik basieren. Nähere Informationen zu der Funktion und den Bauformen dieser Sensoren sind beispielsweise in [3] zu finden. Kameras liefern ein 2-D-Abbild der 3-D-Szene in Form von hoch aufgelösten Grauwertoder Farbbildern, aus denen bei genügendem Kontrast oder Unterschieden in der Textur einzelne Objekte mit Methoden der Bildverarbeitung extrahiert werden können. Eine Bestimmung der Objektentfernung ist mit Mono-Kameras allerdings nur mit oft zu Fehlern führenden Annahmen wie einer ebenen Fahrbahn möglich. Stereo-Kameras erlauben zwar über das Disparitätsbild auch eine Bestimmung der Objektentfernung, die Genauigkeit sinkt jedoch quadratisch mit zunehmender Entfernung. Bei den heute üblichen Basisabständen der Stereoanordnungen und Auflösung der Kameras sind Messreichweiten bis etwa 50 Meter möglich, ohne dass der Fehler so stark ansteigt, dass Funktionen hieraus keinen Nutzen mehr ziehen könnten.

Radar-, aber auch Lidarsensoren liefern hingegen vergleichsweise sehr genaue und auch hinsichtlich der Messfehler nahezu distanzunabhängige Entfernungsmessdaten. Sie können aber aufgrund der geringeren Winkelauflösung die Konturen, d. h. die Außenabmessungen von Objekten, schlechter erfassen. Dies gilt insbesondere für Radarsensoren. Zudem liefern Radar- und Lidarsensoren keinerlei Texturinformationen. Aufgrund dieser unterschiedlichen Messeigenschaften werden die unterschiedlichen Sensortypen für die Aufgabe der maschinellen Wahrnehmung in der Regel kombiniert verwendet. Man spricht dann von einer Sensordatenfusion.

Mit den fusionierten Sensordaten lassen sich bewegte und statische Objekte, aber beispielsweise auch Fahrbahnmarkierungen grundsätzlich erkennen und physikalisch vermessen. Die möglichen Messdimensionen hängen vom spezifischen Sensor-Setup ab. Typische erfassbare physikalische Messdaten sind die Abmessungen eines Objekts als Quadermodell mit Länge, Breite und Höhe sowie seine Position absolut in der Welt oder relativ zum eigenen Fahrzeug. Im Fall von bewegten Objekten kommen dann noch die Objektgeschwindigkeiten und Objektbeschleunigungen als relativ einfach bestimmbare Zustandsgrößen hinzu. Schwerer und in der Regel sehr unsicher bestimmbar aus externen Sensormessungen ist die Gierrate beziehungsweise die Kursrate anderer Verkehrsteilnehmer. Diese Größen sind ohne Fahrzeug-zu-Fahrzeug-Kommunikation nur für das eigene Fahrzeug zuverlässig ermittelbar. 
Für die spätere Situationsbewertung und Situationsprädiktion ist allerdings nicht nur die physikalische Vermessung der Objekte notwendig, sondern auch die Kenntnis darüber, um welche Objektklasse es sich handelt. Beispielsweise unterscheiden sich ein Fußgänger und ein Motorradfahrer in ihren möglichen Bewegungsfreiheitsgraden und auch in der möglichen Bewegungsdynamik. Markierungsstreifen auf der Fahrbahn können je nach Kontext und Konstellation unterschiedliche Bedeutungen haben. Daher ist es notwendig, auch die semantische Bedeutung der erkannten Objekte aus den Sensordaten oder weiteren Informationsquellen wie einer digitalen Karte zu ermitteln. Dieser Vorgang wird im Zusammenhang mit der maschinellen Wahrnehmung als Klassifikationsschritt bezeichnet, ist aber Bestandteil der maschinellen Wahrnehmung.

Während Menschen sehr schnell und fehlerfrei den visuellen Wahrnehmungen auch semantische Bedeutung zuordnen können, ist dies für die maschinelle Wahrnehmung nach dem heutigen Stand der Technik noch eine vergleichsweise schwierige Aufgabe. Bekannte Klassifikationsverfahren basieren immer auf mehr oder weniger komplexen Modellen erwarteter Objektklassen, die entweder aus Beispielen automatisiert gelernt oder manuell vorgegeben werden. Diese Modelle weisen dann möglichst diskriminierende, mit den verfügbaren Sensoren erfassbare Merkmale auf, sodass zwischen den vorkommenden Objektklassen unterschieden werden kann. Es wird aber auch deutlich, dass vorab nicht trainierte Objektklassen mit heute bekannten Verfahren auch nicht semantisch identifiziert werden können. Aufgrund der signifikant höheren Leistungsfähigkeit haben sich lernende Klassifikationsverfahren heute weitgehend durchgesetzt.

Eine maschinelle Wahrnehmung mit semantischen Informationen ist im Kontext der Fahrerassistenzsysteme und des automatisierten Fahrens nur deshalb technisch möglich, weil der Verkehrsraum gut strukturiert und auf wenige Objektklassen beschränkt ist. Zudem ist für die Situationserkennung und Situationsprädiktion nur eine grobe Klassenunterscheidung relevant. Nach dem heutigen Stand der Technik reicht es aus, unter den bewegten Objekten zwischen den Klassen Fußgänger, Radfahrer, Personenkraftwagen und Lastkraftwagen bzw. Bussen unterscheiden zu können. Hinzu kommen noch stationäre Hindernisse, die jedoch meist zusammen mit den nicht klassifizierbaren Objekten einer Restklasse hinzugefügt werden.

Für die korrekte Zuordnung der klassifizierten Objekte zur Verkehrsinfrastruktur ist es ferner notwendig, auch Fahrstreifenmarkierungen, Sperrflächen, Haltelinien, Lichtsignalanlagen und Verkehrsschilder zuverlässig mit korrekter semantischer Bedeutung zu erkennen. Da diese komplexe Klassifikationsaufgabe heute noch nicht mit der notwendigen Zuverlässigkeit möglich ist, werden nach dem Stand der Technik unterstützend hochgenaue und umfassend attributierte digitale Karten verwendet. Hieraus kann das automatisierte Fahrzeug bei bekannter eigener Position die im Sensorsichtfeld zu erwartenden stationären Objekte sowie Markierungen inklusive semantischer Bedeutung entnehmen. Die Sensorik muss dann nur noch das Vorhandensein der Objekte verifizieren.

Ein Nachteil dieser Vorgehensweise ist es, dass zum einen eine hochgenaue Lokalisation des eigenen Fahrzeugs notwendig ist, für die Standard-GPS-Verfahren nicht ausreichen, und zum anderen die Karte immer aktuell sein muss. Aus diesem Grund wird man zukünf- 


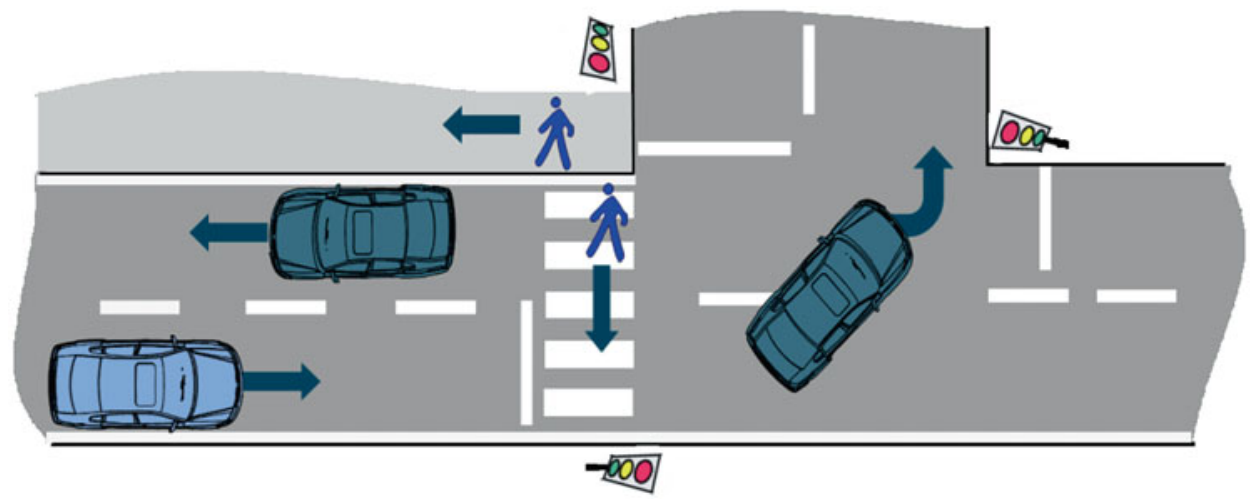

Abb. 20.2 Prinzipbild der objektbasierten Fahrumgebungsrepräsentation. Es werden alle relevanten Objekte erkannt, klassifiziert und der Infrastruktur korrekt zugeordnet

tig versuchen technische Lösungen zu entwickeln, die keine hochgenaue und aktuelle Karte mehr benötigen.

\subsubsection{Ausprägung von Umgebungsmodellen}

Die maschinelle Wahrnehmung mündet in ein Fahrumgebungsmodell, wobei man objektbasierte und gitterbasierte Repräsentationsformen unterscheidet. Beide Repräsentationsformen sind grundsätzlich kombinierbar.

Unter einem objektbasierten Fahrzeugumfeldmodell versteht man eine dynamische Datenstruktur, in der alle relevanten Objekte und Infrastrukturelemente in der Nähe des eigenen Fahrzeugs korrekt in Ort und Zeit in einem gemeinsamen Bezugssystem enthalten sind. Die Erfassung und zeitliche Verfolgung der Objekte und Infrastrukturelemente erfolgen wie oben erläutert fortlaufend durch geeignete, in der Regel fusionierte bordeigene Sensoren wie Kameras und Radare sowie die zusätzliche Nutzung hochgenauer digitaler Karten. Abb. 20.2 zeigt beispielhaft Komponenten, die eine Fahrumgebungsrepräsentation enthalten kann.

Welche Objekte und Strukturelemente für das automatisierte Fahren relevant sind, hängt maßgeblich von der zu bewältigenden Fahraufgabe ab, deren Komplexität beginnend mit einfachen Autobahnszenarien, über Landstraßen bis hin zum innerstädtischen Verkehr stark zunimmt. Bei der objektbasierten Repräsentation werden alle für die Repräsentation relevanten anderen Verkehrsteilnehmer, die relevanten Infrastrukturelemente sowie das eigene Fahrzeug selbst jeweils durch ein eigenes dynamisches Objektmodell, in der Regel ein zeitdiskretes Zustandsraummodell, beschrieben. Dessen Zustände wie Position, Geschwindigkeit oder auch 2-D/3-D-Objektausdehnung werden schritthaltend mit den Sensormessungen fortlaufend aktualisiert. Ferner erfolgt eine fortlaufende Erfassung der Fahrbahnmarkierungen sowie der Verkehrszeichen und des Status der Lichtsignalanlagen. 


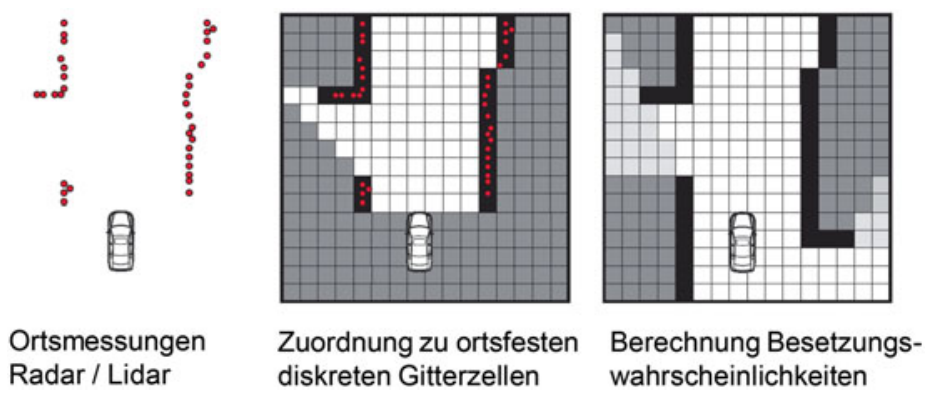

Abb. 20.3 Prinzipbild des Aufbaus einer rasterbasierten Fahrumgebungsrepräsentation. Hier sind im einfachsten Fall nur statische Hindernisse enthalten.

Eine gitterbasierte Darstellung verwendet Rasterkarten, um die Umwelt ortsfest in gleich große Zellen einzuteilen. Das Fahrzeug bewegt sich über dieses ortsfeste 2-D- oder 3-D-Gitter, und die bordautarke Sensorik liefert dann nur Informationen, ob spezifische Zellen frei sind und damit frei befahren werden können oder ob sich in der jeweiligen Zelle ein Hindernis befindet. Zudem kann auch der Zustand modelliert werden, dass über Zellen keine Informationen vorliegen. Diese Art der Darstellung eignet sich vornehmlich zur Repräsentation statischer Szenarien und statischer Hindernisse. Sie benötigt keinerlei Modellhypothesen über die zu erwartenden Objektklassen und ist daher als sehr robust gegen Modellfehler einzustufen. Abb. 20.3 zeigt die grundsätzliche Vorgehensweise. Mehr Informationen zu gitterbasierten Repräsentationen ist [4], [5], [6], [7] und [8] zu entnehmen.

\subsection{Methoden zum Umgang mit Unsicherheiten der maschinellen Wahrnehmung}

\subsubsection{Unsicherheitsdomänen}

Wie in Abschn. 20.2 beschrieben, umfasst die maschinelle Wahrnehmung unterschiedliche Aufgabenumfänge. Diese sind zum einen die Detektion von statischen und dynamischen Objekten und ihre möglichst genaue physikalische Vermessung, zum anderen aber auch die Zuordnung der korrekten semantischen Bedeutung der detektierten Objekte. Entsprechend diesen Aufgaben existieren für die maschinelle Wahrnehmung die folgenden drei Unsicherheitsdomänen:

\section{Zustandsunsicherheit}

Die Zustandsunsicherheit beschreibt die Unsicherheit in den physikalischen Messgrößen wie Größe, Position und Geschwindigkeit und ist eine direkte Folge der bei Sensoren prinzipiell nicht vermeidbaren Messfehler. 
2. Existenzunsicherheit

Die Existenzunsicherheit beschreibt die Unsicherheit darüber, ob ein von der Sensorik erkanntes und in die Umgebungsrepräsentation übernommenes Objekt überhaupt real existiert. Fehler dieser Art können durch Unzulänglichkeiten in den Signalverarbeitungsalgorithmen oder durch Fehlmessungen der Sensoren selbst entstehen.

3. Klassenunsicherheit

Hiermit ist die Unsicherheit hinsichtlich der korrekten semantischen Zuordnung gemeint, die durch Unzulänglichkeiten der Klassifikationsverfahren bzw. nicht ausreichend gute Messdaten verursacht werden können.

Für die Anwendung des automatisierten Fahrens ist es notwendig, die Unsicherheiten bzw. Fehler in den verschiedenen Domänen zuverlässig zu erkennen und, falls möglich, sogar prädizieren zu können. Methodisch werden zur Behandlung von Unsicherheiten nach dem Stand der Technik fast ausnahmslos Verfahren basierend auf der Bayes-Theorie [10], [11], [12] oder ihrer Verallgemeinerung, der Dempster-Shafer-Theorie [16], verwendet. Sie besitzen den Vorteil, dass sie eine durchgehend probabilistische und daher weitgehend heuristikfreie Behandlung der Unsicherheitsdomänen erlauben.

Die o.g. Unsicherheitsdomänen beziehen sich im engeren Sinne zunächst nur auf die bordeigene Sensorik. Es lassen sich allerdings auch Fehler in Informationen aus einer digitalen Karte oder den durch Car2x-Kommunikation gewonnenen Daten entsprechend kategorisieren. Gerade die zuletzt genannte Car2x-Kommunikation birgt zwar durch mögliche variable Latenzzeiten bei der Übertragung und eventuell nicht genau bekannte Unsicherheitsbewertungen der Sendequellen zusätzliche Fehlereinflüsse. Die Auswirkungen lassen sich jedoch wieder den drei genannten Unsicherheitsdomänen zuordnen, sodass hierauf nicht weiter eingegangen wird.

\subsubsection{Zustandsunsicherheit}

Die Zustandsunsicherheit eines detektierten Objektes wird gemäß der Bayes-Theorie durch eine Wahrscheinlichkeitsdichtefunktion beschrieben, anhand derer der wahrscheinlichste Gesamt- bzw. Einzelzustand sowie mit gewisser Wahrscheinlichkeit auch mögliche Variationen hiervon bestimmt werden können. Im Fall einer mehrdimensionalen, normalverteilten Wahrscheinlichkeitsdichtefunktion ist die Zustandsunsicherheit durch eine Kovarianzmatrix vollständig repräsentiert.

Bei der Schätzung statischer Größen wie beispielsweise die Fahrzeugabmessungen kann deren Zustandsunsicherheit durch wiederholte Messungen immer weiter verringert werden. Der Schätzwert auf Basis der verfügbaren Messungen konvergiert gegen den wahren Wert, falls kein systematischer Sensorfehler, beispielsweise in Form eines Offsets, vorliegt. Bei der Schätzung von dynamischen, zeitveränderlichen Zuständen wie der Objektposition oder Objektgeschwindigkeit ist aufgrund der Bewegung des Objektes zwischen den Messzeitpunkten die Konvergenz gegen einen wahren Wert nicht mehr gegeben. Bei der Bewer- 
tung der Güte der Zustandsschätzung wird daher gefordert, dass der Fehler im Mittel null ist und die Unsicherheit möglichst gering.

Das grundlegende Verfahren zur Behandlung von Zustandsunsicherheiten ist das allgemeine Bayes-Filter [10]. Bei ihm werden der geschätzte Zustand eines Objektes und die dazugehörige Unsicherheit durch eine mehrdimensionale Wahrscheinlichkeitsdichtefunktion $p$ (engl. probability density function, PDF) repräsentiert:

$$
p_{k+1}\left(x_{k+1} \mid Z_{1: k+1}\right) \text {. }
$$

Sie hängt allgemein von allen bis zum Zeitpunkt $k+1$ vorhandenen Messungen $Z_{1: k+1}=\left\{z_{1}, \ldots, z_{k+1}\right\}$ ab. Dies wird durch die gewählte Schreibweise einer bedingten Wahrscheinlichkeit ausgedrückt, d. h., die Wahrscheinlichkeit für den Zustand des Systems $x$ ist bedingt durch die Messungen $Z$.

Das Bewegungsmodell eines durch die Sensoren erfassten Objektes für den Zeitraum zwischen zwei aufeinanderfolgenden Messungen ist durch eine Bewegungsgleichung der Form

$$
x_{k+1 \mid k}=f\left(x_{k}\right)+v_{k}
$$

zu beschreiben, wobei $v_{k}$ eine additive Störgröße darstellt, die mögliche Modellfehler repräsentiert. Die Bewegungsgleichung drückt aus, in welchem Zustand wie Ort, Geschwindigkeit und Bewegungsrichtung sich das Objekt zum nächsten Zeitpunkt wahrscheinlich befindet. Alternativ kann diese Bewegungsgleichung auch durch eine Markov-Übergangswahrscheinlichkeitsdichte ausgedrückt werden:

$$
f_{k+1 \mid k}\left(x_{k+1} \mid x_{k}\right) \text {. }
$$

Die Markov-Übergangswahrscheinlichkeitsdichte ist letztendlich nur eine andere mathematische Schreibweise für dieselben Modellannahmen. Um die Gleichungen praktisch berechenbar zu halten, ist es üblich, eine Markov-Eigenschaft erster Ordnung vorauszusetzen. Diese Eigenschaft sagt vereinfachend aus, dass der zukünftige Zustand eines Systems nur vom zuletzt bekannten Zustand und der aktuellen Messung abhängt, nicht aber von der gesamten Historie von Messungen und Zuständen. Die Markov-Eigenschaft erster Ordnung ist damit eine vorausgesetzte Systemeigenschaft.

Im konkreten Fall hängt der prädizierte Zustand $x_{k+1}$ des Objektes vor dem Vorliegen der neuen Messung dann nur noch vom zuletzt ermittelten Zustand $x_{k}$ ab, da dieser implizit die gesamte Messhistorie $Z_{1: k}=\left\{z_{1}, \ldots, z_{k}\right\}$ enthält.

Die Vorhersage des aktuellen Objektzustandes $x_{k}$ bis zum nächsten Messzeitpunkt $k+1$ erfolgt schließlich unter Berücksichtigung der Modellannahmen anhand der Chapman-Kolmogorov-Gleichung

$$
p_{k+1 \mid k}\left(x_{k+1} \mid x_{k}\right)=\int f_{k+1 \mid k}\left(x_{k+1} \mid x_{k}\right) p_{k}\left(x_{k}\right) d x_{k} .
$$

Dies wird als Prädiktionsschritt des Bayes-Filters bezeichnet. 
Der Messprozess der Sensoren lässt sich allgemein durch eine Messgleichung der Form

$$
z_{k+1 \mid k}=h_{k+1}\left(x_{k+1}\right)+w_{k+1}
$$

beschreiben. Die Messfunktion $h($.$) beschreibt, wie Messungen und Zustandsgrößen zu-$ sammenhängen. Kann beispielsweise eine Zustandsgröße direkt gemessen werden, handelt es sich bei $h\left(\right.$.) um eine Eins-zu-eins-Abbildung. Die stochastische Störgröße $w_{k+1}$ repräsentiert hierbei einen möglichen Messfehler. Eine alternative mathematische Repräsentation der Messgleichung ist die Likelihood-Funktion

$$
g\left(z_{k+1} \mid x_{k+1}\right)
$$

Bei Vorliegen der aktuellen Messungen $z_{k+1}$ wird die Wahrscheinlichkeitsdichtefunktion des Objektzustandes aktualisiert. Die Berechnung der aktuellen Schätzung des Zustands erfolgt anhand der Bayes-Formel

$$
p_{k+1}\left(x_{k+1} \mid z_{k+1}\right)=\frac{g\left(z_{k+1} \mid x_{k+1}\right) p_{k+1 \mid k}\left(x_{k+1} \mid x_{k}\right)}{\int g\left(z_{k+1} \mid x_{k+1}\right) p_{k+1 \mid k}\left(x_{k+1} \mid x_{k}\right) d x} .
$$

Dieser zweite Schritt zum Einbringen der aktuellen Messung wird als Innovationsschritt bezeichnet.

Das durch Prädiktionsschritt und Innovationsschritt kurz beschriebene rekursive Schätzverfahren wird als allgemeines Bayes-Filter bezeichnet, worauf alle heute üblichen Methoden und Implementationen der stochastischen Zustandsschätzung basieren. Neben den Prozess- und Messgleichungen benötigt das Verfahren nur eine Apriori-PDF für den Objektzustand $p_{0}\left(x_{0}\right)$ zum Zeitpunkt $k=0$. Das Filter ist in dieser allgemeinen Form jedoch nicht effizient zu implementieren.

Unter der Annahme von normalverteilten Messsignalen sowie linearen Modellen ermöglicht das Kalman-Filter [9] eine einfache analytische Implementation des allgemeinen Bayes-Filters. Da eine Gauß-Verteilung durch ihre ersten beiden statistischen Momente, d.h. den Mittelwert sowie die zugehörige Kovarianzmatrix, vollständig beschrieben ist, stellt die zeitliche Filterung der Momente eine mathematisch exakte Lösung dar. Eine Anwendung des Kalman-Filters auf Systeme mit nichtlinearen Prozess- oder Messgleichungen lässt sich anhand des Extended-Kalman-Filters (EKF) [12] sowie des UnscentedKalman-Filters (UKF) [15] realisieren. Während das EKF die Systemgleichungen unter Nutzung einer Taylorreihen-Approximation linearisiert, ist das Ziel des UKF eine stochastische Approximation anhand sogenannter Sigma-Punkte [15].

Unabhängig von der konkreten Implementation ist allen auf dem allgemeinen BayesFilter basierenden Verfahren gemein, dass sie fortlaufend ein probabilistisches Maß für die Unsicherheit der aus den Sensordaten bestimmten physikalischen Größen liefern. Hierdurch lassen sich Ausfälle von Sensoren, aber auch eine Degeneration der Leistungsfähigkeit einzelner Sensoren zuverlässig erkennen. Weichen beispielsweise Messdaten einzelner 
Sensoren signifikant, d. h. außerhalb der statistisch zu erwartenden Schwankungsbreite, ab, liegt eine entsprechende Leistungsminderung vor.

Es bleibt jedoch festzuhalten, dass eine Einschränkung der Sensorfunktion nur erkannt werden kann, nachdem sie aufgetreten ist. Außer Trendaussagen bei langsamer Degeneration ist keinerlei Vorhersage der zukünftigen Wahrnehmungsleistung bezogen auf die Zustandsunsicherheit möglich.

\subsubsection{Existenzunsicherheit}

Für die Realisierung des automatisierten Fahrens ist die Existenzunsicherheit mindestens genauso relevant wie die Zustandsunsicherheit. Sie drückt aus, mit welcher Wahrscheinlichkeit das Objekt in der Fahrumgebungsrepräsentation auch wirklich einem realen Objekt entspricht. Eine Notbremsung eines automatisierten Fahrzeugs sollte beispielsweise nur im Falle einer sehr hohen Existenzwahrscheinlichkeit eines erkannten Hindernisses ausgelöst werden.

Während die Schätzung von Zustandsunsicherheiten nach dem Stand der Technik durch Methoden der Bayes-Schätzung theoretisch fundiert erfolgt, wird die Existenzwahrscheinlichkeit in heutigen Systemen noch meist aufgrund eines heuristischen Qualitätsmaßes bestimmt. Ein Objekt gilt als bestätigt, wenn das Qualitätsmaß einen sensor- und anwendungsabhängigen Schwellwert überschreitet. Die Qualitätsmaße basieren beispielsweise auf der Anzahl der Messungen, die das Objekt bestätigt haben, oder einfach auf der Zeitspanne zwischen Initialisierung des Objektes und dem aktuellen Zeitpunkt. Häufig wird auch die Zustandsunsicherheit des Objektes (s. Abschn. 20.3.2) zur Validierung verwendet.

Ein theoretisch besser fundierter Ansatz ist die Schätzung einer wahrscheinlichkeitsbasierten Existenzwahrscheinlichkeit. Hierfür ist zunächst eine Definition der spezifischen Objektexistenz notwendig. Während in manchen Anwendungen sämtliche realen Objekte als existent betrachtet werden, kann die Objektexistenz auch auf die in der aktuellen Anwendung relevanten Objekte eingeschränkt werden. Des Weiteren ist eine Einschränkung auf die mit dem aktuellen Sensor-Setup auch detektierbaren Objekte möglich. Im Gegensatz zu einem Schwellwertverfahren ermöglicht diese Bestimmung der Existenzwahrscheinlichkeit jedoch eine wahrscheinlichkeitsbasierte Interpretationsmöglichkeit. Beispielsweise bedeutet eine Existenzwahrscheinlichkeit von 90 Prozent, dass die Messhistorie sowie das Bewegungsmuster des Objektes mit einer Wahrscheinlichkeit von 90 Prozent von einem realen Objekt erzeugt wurden. Folglich kann die Handlungsplanung des automatisierten Fahrzeugs diese Wahrscheinlichkeiten bei der Bewertung von Handlungsalternativen verwenden.

Ein bekannter Algorithmus zur Berechnung einer Existenzwahrscheinlichkeit stellt das ebenfalls auf dem allgemeinen Bayes-Filter aufbauende Joint-Integrated-ProbabilisticData-Association- (JIPDA-) Verfahren dar, das erstmals 2004 von Musicki [13] vorgestellt wurde. Es verwendet hierfür zusätzlich die als bekannt vorausgesetzten Detektions- und Falschalarmwahrscheinlichkeiten der Sensoren. 


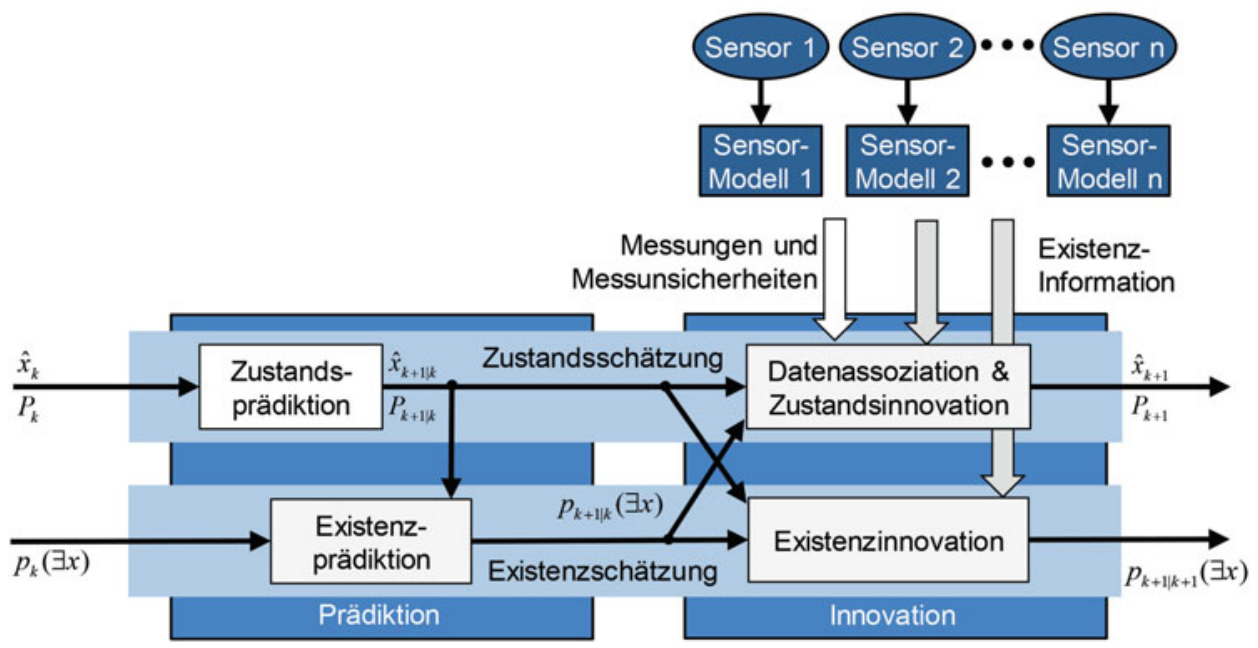

Abb. 20.4 Struktur eines JIPDA-Filters als Verkopplung zweier Markov-Ketten

Die Berechnung der aktuellen Objektexistenzwahrscheinlichkeit erfolgt analog zur Aktualisierung des Zustands im Kalman-Filter in einem Prädiktions- und einem Innovationsschritt. Die Existenzprädiktion erfolgt anhand eines Markov-Modells erster Ordnung. Die prädizierte Existenz eines Objektes ist durch die Markov-Kette

$$
p_{k+1 \mid k}(\exists x)=p_{S} p_{k}(\exists x)+p_{B} p_{k}(\nexists x)
$$

gegeben, wobei die Wahrscheinlichkeit $p_{s}$ die Persistenzwahrscheinlichkeit des Objektes darstellt und $p_{B}$ die Wahrscheinlichkeit für das Eintreten eines Objektes in den Sensorerfassungsbereich. Folglich ist die Wahrscheinlichkeit für das Verschwinden eines Objektes gegeben durch $1-p_{s}$. Im Innovationsschritt wird die Aposteriori-Existenzwahrscheinlichkeit $p_{k+1}(\exists x)$ berechnet. Sie hängt im Wesentlichen davon ab, wie viele aktuelle Messungen die Existenz des Objektes bestätigen.

Da die Persistenzwahrscheinlichkeit eines Objektes vom aktuellen Objektzustand abhängt und die Aposteriori-Existenzwahrscheinlichkeit wiederum von den Datenassoziationen, kann das JIPDA-Filter als die in Abb. 20.4 dargestellte Verkopplung zweier Markov-Ketten interpretiert werden. Die obere Markov-Kette stellt die aus dem Kalman-Filter bekannte Zustandsprädiktion und Innovation dar, während die untere Markov-Kette die Prädiktion und Innovation der Existenzwahrscheinlichkeit repräsentiert. Für Details zum JIPDA-Verfahren und seine spezifische Formulierung hinsichtlich der Anwendungen im Automotive-Bereich sei beispielsweise auf [14] verwiesen. Aktuelle und erst in den letzten Jahren entwickelte Multi-Objekttracking-Verfahren erlauben ebenfalls eine integrierte objektspezifische Existenzschätzung. Für weitere Informationen hierzu sei auf [10], [17], [18] und [19] verwiesen. 
Hinsichtlich des funktionalen Verhaltens der Existenzschätzung gelten dieselben Einschränkungen wie bei der Zustandsschätzung. Es wird fortlaufend ein probabilistisches Maß für die spezifische Existenz des Objekts geliefert. Sensorausfälle während des Betriebs können daher auch in dieser Unsicherheitsdomäne zuverlässig erkannt werden. Eine Vorausschau auf eine künftige Leistungsfähigkeit ist allerdings hier ebenfalls nicht möglich.

\subsubsection{Klassenunsicherheit}

Klassifikationsverfahren zur Bestimmung der Objektklasse, also das Bestimmen semantischer Informationen, sind sehr sensorspezifisch aufgebaut. Aufgrund des signifikant höheren Informationsgehaltes überwiegen im Bereich der Klassifikation bildbasierte Verfahren. Grundsätzlich unterscheidet man lernende Verfahren, bei denen der Klassifikator aufgrund von Positiv- und Negativbeispielen offline trainiert wird und der dann im Onlinebetrieb die trainierten Objektklassen mehr oder weniger gut erkennen kann. Die im Training verwendeten Merkmale werden entweder vorgegeben oder auch selbst im Lernprozess implizit generiert. Methodisch haben sich bei den lernenden Verfahren zwei grundsätzliche Vorgehensweisen etabliert. Zum einen sind dies kaskadierte Verfahren nach Viola und Jones [20] oder Methoden basierend auf verschiedenen Neuronalen Netzen [21], [22].

Eine eher klassische, aber ebenfalls noch übliche Vorgehensweise ist es, aus Sensordaten möglichst zahlreiche, für die unterschiedlichen Klassen diskriminierende deterministische Merkmale wie Länge, Breite oder Geschwindigkeit festzulegen und hierfür die klassenspezifischen statistischen Schwankungsbereiche zu ermitteln. Der Mittelwert der Einzelmerkmale wird inklusive Schwankungsbreite beispielsweise durch eine Normalverteilung approximiert. Anschließend erfolgt auf Basis der aktuellen Messwerte und der bekannten Merkmalverteilungen die Bestimmung der nach im Bayes'schen Sinne wahrscheinlichsten Klasse. Sollen unterschiedliche Sensoren kombiniert verwendet werden, die jeweils nur einzelne Merkmale des Gesamtsets erfassen können, bietet sich die Dempster-ShaferTheorie [16] zur Klassenbestimmung an, da sie es erlaubt, auch „Nichtwissen“ zu berücksichtigen. Diese Verfahren sind in der Regel allerdings weniger leistungsfähig als lernende Verfahren, sodass sie vermutlich weiter an Bedeutung verlieren werden.

Nachteilig bei allen genannten Klassifikationsverfahren ist, dass keine theoretisch fundierten Wahrscheinlichkeiten für die augenblickliche Güte der Klassifikation bestimmt werden können. Hierzu existiert zurzeit keine umfassende theoretische Basis. Als Ausgabe der Klassifikatoren dient zurzeit lediglich ein individuelles Zuverlässigkeitsmaß, das auf den Wertebereich von null bis eins normiert werden kann. Eine Wahrscheinlichkeit im engeren Sinne stellt es nicht dar, sodass unterschiedliche Algorithmen diesbezüglich auch nicht vergleichbar sind. Bildbasierte trainierte Klassifikatoren unterscheiden sich so sehr von merkmalbasierten Verfahren aus Lidar- oder Radarsensoren, dass deren einheitliche Behandlung nicht einfach möglich sein wird. 
Tab. 20.1 Unsicherheitsdomänen der maschinellen Wahrnehmung und deren methodische Behandlung

\begin{tabular}{|c|c|c|c|}
\hline & Zustandsunsicherheit & Existenzunsicherheit & Klassenunsicherheit \\
\hline Ausprägung & $\begin{array}{l}\text { Unsicherheit in den } \\
\text { Zustandsgrößen wie } \\
\text { Objektposition, Objekt- } \\
\text { geschwindigkeit etc. }\end{array}$ & $\begin{array}{l}\text { Unsicherheit, ob ein } \\
\text { von Sensorik erfasstes } \\
\text { Objekt real existiert }\end{array}$ & $\begin{array}{l}\text { Unsicherheit über } \\
\text { Klassenzugehörigkeit } \\
\text { (z.B. Pkw <-> Lkw) }\end{array}$ \\
\hline Ursache & $\begin{array}{l}\text { stochastische Mess- } \\
\text { fehler der verwendeten } \\
\text { Sensorik }\end{array}$ & $\begin{array}{l}\text { Detektionsunsicherhei- } \\
\text { ten einzelner Sensoren, } \\
\text { beispielsweise Kamera } \\
\text { oder Radar }\end{array}$ & $\begin{array}{l}\text { Klassifikationsunsicher- } \\
\text { heiten der Algorithmen / } \\
\text { Limitierungen einzelner } \\
\text { Sensoren }\end{array}$ \\
\hline Modellierung & $\begin{array}{l}\text { probabilistisch, Erwar- } \\
\text { tungswert mit Varianzen/ } \\
\text { Kovarianzen }\end{array}$ & $\begin{array}{l}\text { probabilistisch über } \\
\text { Detektionswahrschein- } \\
\text { lichkeiten }\end{array}$ & $\begin{array}{l}\text { keine durchgehende } \\
\text { Methode, zurzeit vor- } \\
\text { wiegend heuristisch }\end{array}$ \\
\hline Methoden & $\begin{array}{l}\text { geschlossene Theorie } \\
\text { über allgemeines Bayes- } \\
\text { Filter (z. B. Variante } \\
\text { Kalman-Filter) }\end{array}$ & $\begin{array}{l}\text { geschlossene Theorie, } \\
\text { gekoppelt an Schätzung } \\
\text { Zustandsunsicherheit } \\
\text { (z.B. JIPDA-Filter) }\end{array}$ & $\begin{array}{l}\text { merkmalbasiert: } \\
\text { Bayes, Dempster-Shafer, } \\
\text { lernbasiert: } \\
\text { Neuronale Netze, } \\
\text { kaskadierte Verfahren } \\
\text { (Viola und Jones etc.) }\end{array}$ \\
\hline $\begin{array}{l}\text { Prädiktion in } \\
\text { die Zukunft }\end{array}$ & $\begin{array}{l}\text { generell nein, bedingt } \\
\text { möglich über Trend- } \\
\text { aussagen }\end{array}$ & generell nein & generell nein \\
\hline
\end{tabular}

\subsubsection{Zusammenfassende Bewertung}

Aus den Betrachtungen wird deutlich, dass die maschinelle Wahrnehmung drei wesentliche Unsicherheitsdomänen umfasst, nämlich die Zustandsunsicherheit, die Existenzunsicherheit und die Klassenunsicherheit. Alle Domänen haben direkten Einfluss auf die Leistungsfähigkeit der maschinellen Wahrnehmung. Werden die Unsicherheiten zu hoch, wobei funktionsspezifisch festzulegen ist, welche Unsicherheiten noch tolerabel sind, ist keine sichere Führung eines automatisierten Fahrzeugs mehr möglich.

Problematisch ist, dass eine zukünftig höhere Unsicherheit und damit eine größere Fehlerwahrscheinlichkeit nicht zeitlich vorhergesagt werden kann. Die heute bekannten Methoden zur Schätzung von Zustands- und Existenzunsicherheiten erlauben zwar eine aktuelle Einschätzung der Leistungsfähigkeit der maschinellen Wahrnehmung, eine Degeneration der Leistungsfähigkeit einzelner Sensoren oder gar ein Ausfall von Komponenten kann aber prinzipbedingt nicht vorhergesagt werden. Lediglich Trendaussagen sind möglich. Die folgende Tab. 20.1 fasst die Ergebnisse noch einmal zusammen. 


\subsection{Folgerungen für die maschinelle Wahrnehmungsleistungsprädiktion}

Wie in den vorherigen Abschnitten ausgeführt und begründet, kann die zukünftige Entwicklung der maschinellen Wahrnehmungsleistung eines automatisierten Fahrzeugs aus grundlegenden Überlegungen heraus nicht mit ausreichender Konfidenz vorausgesagt werden. In keinem Fall kann die Wahrnehmungsleistung für den zur Übergabe der Fahraufgabe an den Menschen notwendigen Rückgabezeitraum von fünf bis zehn Sekunden unter allen Umständen sicher vorhergesagt werden, wie sie beim hochautomatisierten Fahren als Rückfalloption vorgesehen ist. Ein vollautomatisiertes Fahrzeug müsste autonom einen eigensicheren Zustand einnehmen können, wozu in einigen Fällen noch ein längerer Zeitraum erforderlich wäre als im Übernahmefall durch einen Fahrer. Obwohl es sicher einige Optionen der Prädiktion der zukünftigen Einschränkung der Wahrnehmungsleistungsfähigkeit aufgrund äußerer Bedingungen wie beispielsweise eine bevorstehende Kamerablendung durch eine tief stehende Sonne, Einschränkungen der Sensoren durch einsetzenden Regen, Schnee oder Nebelbänke gibt, sind dies spezielle Szenarien, die außerdem eine extrem robuste Kontextinformation benötigen. Somit ist eine Prädiktion der Wahrnehmungsleistungsfähigkeit prinzipbedingt keine generelle Option zur Gewährung der notwendigen Sicherheit beim automatisierten Fahren.

Wie oben ausgeführt, existieren jedoch bereits theoretisch fundierte Methoden und Verfahren, um die aktuelle maschinelle Wahrnehmungsleistung kontinuierlich zu überwachen und Systemausfälle sowie Degradationen einzelner Komponenten zeitnah und sicher erkennen zu können. Konzeptionell müssten daher maschinelle Wahrnehmungssysteme so ausgelegt werden, dass eine sensorische Redundanz vorhanden ist, die eine ausreichende Restfunktion der Wahrnehmungsleistung entweder bis zur Übergabe an den Fahrer oder im Fall des vollautomatisierten Fahrzeugs bis zum Erreichen eines eigensicheren Zustands bei Ausfall einzelner Komponenten gewährleistet. Ein komplettes Versagen der maschinellen Wahrnehmung darf daher nicht auftreten.

Derartige Redundanzen bieten grundsätzlich Multisensorsysteme, die Informationen verschiedener Sensoren und Sensorprinzipien parallel nutzen und fusionieren. Sind beispielsweise Radar- und Lidarsensoren verbaut, so liefern beide Entfernungsmessdaten, allerdings in unterschiedlicher Qualität und in einem unterschiedlichen Sensorerfassungsbereich. Auch die Witterungsabhängigkeiten der Sensorprinzipien sind unterschiedlich. Aufgrund der Ähnlichkeit der Messdaten können sie sich jedoch gegenseitig stützen bzw. bei geringer Einbuße der Messqualität des Gesamtsystems auch gegenseitig bei Ausfall einer Komponente kompensieren. Erst durch diese Nutzung unabhängiger Sensorprinzipien wird es zudem möglich sein, die im Rahmen der funktionalen Sicherheit für das automatisierte Fahren notwendige höchste Sicherheitsstufe gemäß des Automotive Safety Integrity Level (ASIL D) zu erreichen.

Bei Kameras kann ebenfalls leicht eine Redundanz vorgesehen bzw. hergestellt werden. Fällt beispielsweise eine Kamera eines Stereokamerasystems aus, so steht für Klassifikationsaufgaben und die Erkennung von Straßenmarkierungen noch die zweite Kamera des 
Stereosystems zur Verfügung. Lediglich eine Entfernungsschätzung ist dann aus Stereodaten nicht mehr verfügbar und müsste beispielsweise durch Lidar- oder Radarsensoren kompensiert werden. Voraussetzung für diese Redundanz ist natürlich, dass die Verarbeitungshardware und grundlegende Software der Einzelkameras unabhängig, d. h. redundant ausgelegt sind. Alternativ könnte natürlich auch eine weitere Monokamera inklusive eigener Verarbeitungshardware und -software verbaut werden. Durch derartige Redundanzkonzepte kann somit auch bei Ausfall einzelner Komponenten immer eine Mindestwahrnehmungsleistung des automatisierten Fahrzeugs aufrechterhalten werden.

Die automatisierte Fahrzeugführung auf Bahnebene basiert auf der aktuellen maschinellen Wahrnehmung bzw. der darauf aufbauenden Prädiktion der aktuellen Verkehrssituation. Letztere erfolgt nach dem Stand der Technik im Wesentlichen durch eine einfache Prädiktion des aktuellen Bewegungsverhaltens der Objekte in die Zukunft. Aufgrund der Vielzahl der möglichen und nicht voraussehbaren Ereignisse, insbesondere reaktiver Aktionen anderer Verkehrsteilnehmer, steigen die Unsicherheiten der Situationsprädiktion nach etwa zwei bis drei Sekunden so stark an, dass hierauf keine verlässliche Trajektorienplanung mehr möglich ist. Die Situationsprädiktion kann daher auch nicht den Rückgabezeitraum zum Fahrer beim hochautomatisierten Fahren oder das Erreichen eines eigensicheren $\mathrm{Zu}-$ stands beim vollautomatisierten Fahren sicher überbrücken, falls die maschinelle Wahrnehmung das Fahrzeugumfeldmodell nicht mehr ständig aktualisiert.

Der Mensch ist aufgrund seiner Fahrerfahrung allerdings auch in der Lage, die Gesamtsituation für etwa zwei bis drei Sekunden in die Zukunft mit gewisser Sicherheit vorherzuahnen [23]. Da der Mensch seine Umgebung aber quasikontinuierlich wahrnimmt und interpretiert, reicht dieser kurze Vorhersagehorizont völlig aus, adäquat und deeskalierend in nahezu allen Situationen zu reagieren und Unfälle in aller Regel zu vermeiden. Dies sollte also auch für automatisierte Fahrzeuge möglich sein, wobei hier natürlich zusätzlich Latenzen sowie Unsicherheiten in der Wahrnehmung zu berücksichtigen sind. Voraussetzung ist, wie oben erwähnt, eine garantierte Mindestleistungsfähigkeit der maschinellen Wahrnehmung.

Wesentlich für die Gesamtfunktion ist es allerdings, dass sich das automatisierte Fahrzeug erst gar nicht in eine technisch unlösbare Situation bringt. Die zulässige Kritikalität der Situation muss dabei immer der aktuellen maschinellen Wahrnehmungsleistung entsprechen. Es sind hierbei insbesondere auch plötzlich auftretende Ausfälle und damit eine spontane Reduktion der maschinellen Wahrnehmungsleistung zu berücksichtigen. Innerhalb der einigermaßen verlässlichen Prädiktionszeit für die Situationsentwicklung von zwei bis drei Sekunden muss das automatisierte Fahrzeug dann in der Lage sein, sein Fahrverhalten der geänderten maschinellen Wahrnehmungsleistung anzupassen. Ein einfaches Beispiel wäre das Fahren auf einem Fahrstreifen. Verringert sich durch technische Ausfälle oder Witterungseinflüsse die Sensorreichweite, muss das Fahrzeug in der Lage sein, seine Geschwindigkeit innerhalb der Gültigkeit der Prädiktion der aktuellen Situation anzupassen, was ein sicher lösbares technisches Problem darstellt.

Während diese einfache Situation leicht beschreibbar und untersuchbar ist, ist allgemein heute nicht bekannt, wie es zu kritischen Situationen kommt und was im Hinblick auf die 
technische Leistungsfähigkeit eines automatisierten Fahrzeugs überhaupt als kritisch zu bewerten ist. Für die Absicherung automatisierter Fahrzeuge gewährleistet das Abfahren eines vordefinierten Kilometerumfangs in jedem Fall nicht, dass der dadurch entstehende Datensatz alle möglichen kritischen Situationsentwicklungen (Episoden) enthält. Folglich ist eine Absicherung der Funktionssicherheit auf diese Art und Weise nicht möglich, unabhängig davon, dass die zum statistischen Nachweis der sehr geringen Fehlerraten notwendigen Kilometerleistungen weder praktisch noch wirtschaftlich realisierbar wären.

Eine mögliche zukünftige Forschungsaufgabe wäre daher die Suche nach einer geeigneten mathematischen Repräsentation beliebiger Episoden, die dann den Raum aller möglichen Episoden aufspannen. Aufbauend auf dieser Beschreibung kann dann beispielsweise durch sogenannte Monte-Carlo-Simulationen der gesamte Episodenraum in kritische und unkritische Teilbereiche strukturiert werden, um hieraus eine Aussage zu notwendigen spezifischen Tests ableiten zu können. Ein möglicher methodischer Ansatz hierzu ist das Rejektion-Sampling, wobei jedes Sample eine vollständige Episode repräsentiert. Ausgehend von Grundepisoden, die sich beispielsweise durch unterschiedliche Fahrbahntypen (ein, zwei oder drei Fahrstreifen pro Richtung, Gegenverkehr) oder die Anzahl der Fahrzeuge im Nahbereich unterscheiden, werden durch statistische Variation der Episodenparameter ähnliche Situationen generiert. Bei einer hinreichenden Anzahl an Samples besteht die Erwartung, den Episodenraum vollständig abzudecken. Jede gezogene Episode wird dabei auf ihre physikalische Realisierbarkeit überprüft und irrelevante Episoden verworfen. Die verbleibenden Episoden werden anschließend dahingehend überprüft, ob sich beispielsweise kritische Zeitlücken oder Abstände zwischen Objekten ergeben. Die Kriterien hierfür sind ebenfalls geeignet festzulegen. Die Identifikation und Priorisierung kritischer Situationen erfolgt durch ein folgendes Clustern im Episodenraum.

Ziel eines solchen Vorgehens wäre es, durch die hierarchische Vorgehensweise eine möglichst vollständige, aber dennoch handhabbare Menge potenziell kritischer Episoden zu bestimmen. Diese werden im Anschluss anhand simulierter Daten hinsichtlich der Beherrschbarkeit durch das hochautomatisierte System bei verschiedenen Stufen der maschinellen Wahrnehmungsleistung untersucht. Beispielsweise könnten für ein SensorSetup im Fahrzeug individuelle Reichweiten, Erfassungswinkel und Detektionsraten modelliert werden, um dann systematisch die Konsequenzen für das Verhalten des Fahrzeugs in kritischen Episoden zu untersuchen. Diese Untersuchung kann zunächst für ein voll funktionsfähiges System und im Anschluss unter der Annahme eines Ausfalls von Einzelkomponenten durchgeführt werden.

Eine weitere offene Forschungsfrage ist die nach einer zuverlässigeren Situationsprädiktion, die unter Nutzung von Kontextinformationen und Hypothesen über das zukünftige Verhalten der Verkehrsteilnehmer prinzipiell längere Prädiktionszeiträume ermöglicht. Eine derartige Vorgehensweise wäre insofern gerechtfertigt, als dass unser gesamter Verkehr auf der Kooperation der Verkehrsteilnehmer beruht. Nachteilig hieran wäre natürlich, dass ein unkooperatives Verhalten oder auch schlicht Fehler anderer Verkehrsteilnehmer dann natürlich nicht erwartet und in die Handlungsplanung eines automatisierten Fahrzeugs mit einbezogen werden können. Insofern erlauben solche Ansätze keine weitreichende 
Erweiterung des sicher prädizierbaren Zeitraums, können aber Planungsalgorithmen dennoch unterstützen. Ferner ist anzumerken, dass manuelle Fahrer auch in vielen Situationen keine Chance haben, geeignet zu reagieren, wenn sich andere Fahrzeugführer nicht regelgerecht verhalten oder unvorhergesehene Fahrfehler begehen. Insofern müssen auch an automatisierte Fahrzeuge sicher keine zu übertriebenen Anforderungen gestellt werden. Dies ist aber natürlich eine Frage des gesellschaftlichen Konsenses hinsichtlich des zulässigen Gefährdungspotenzials einer neuen Technologie.

\subsection{Zusammenfassung}

Die existierenden Methoden der Zustands- und Existenzschätzung basieren auf einer geschlossenen, fundierten Theorie und erlauben schritthaltend eine zuverlässige Bewertung der aktuellen Güte der maschinellen Wahrnehmungsleistung. Hiermit ist es möglich, Komplettausfälle einzelner Sensoren sowie eine schleichende Degeneration in der Sensorik und/ oder Wahrnehmung festzustellen.

Die Verfahren erlauben jedoch keine Vorhersage der zukünftigen Wahrnehmungsleistung, lediglich ein lineares Fortschreiben von erkannten Trends ist denkbar. Die Zuverlässigkeit und Güte der Bewertung der maschinellen Wahrnehmungsleistung ist von verfügbaren Sensormodellen abhängig, insbesondere von Fehlermodellen, die sensor- und herstellerspezifisch sind. Die Wahrnehmungssysteme allein besitzen keine ausreichende Prädiktionsfähigkeit, die einen Zeithorizont zwischen fünf und zehn Sekunden sicher abdecken könnte, so wie er heute für den Rückgabefall des hochautomatisierten Systems an den Fahrer angesehen wird. Dies ist allerdings für ein sicheres Verhalten eines automatisierten Fahrzeugs vermutlich auch gar nicht notwendig. Entscheidend für die Beherrschbarkeit von Situationen beim automatisierten Fahren sind ausreichend viele, physikalisch umsetzbare und sichere Handlungsoptionen des automatisierten Fahrzeugs. Diese sind im Wesentlichen durch die räumliche Nähe von begrenzenden Objekten zum eigenen Fahrzeug und den verfügbaren, befahrbaren Freiraum bestimmt, sodass räumliche Nähe unter Einbeziehung von Unsicherheiten in der Wahrnehmung und die Zahl der physikalisch möglichen, sicheren Handlungsoptionen in Kennzahlen zur Bewertung einer Kritikalität einfließen müssen. Auch muss die aktuell verfügbare maschinelle Wahrnehmungsleistung hierin Berücksichtigung finden. Derartige ausreichend abgestimmte und theoretisch fundierte Kritikalitätsmaße existieren heute noch nicht.

Eine Situationsprädiktion in die Zukunft über einen Zeitraum von zwei bis drei Sekunden hinaus wird bei rein modellbasierter, probabilistischer Fortschreibung keine Aussage mehr liefern, da jede Entwicklung der Situation möglich ist. Ein möglicher Lösungsansatz und eine zukünftige Forschungsfrage ist die kontextbezogene, hypothesenbasierte zeitliche Fortschreibung einer erkannten und bewerteten Situation. Eine Möglichkeit ist hier, in Anlehnung an das menschliche Verhalten, Situationsepisoden, d.h. reale Entwicklungen aus einem beliebigen Anfangsstand im Fahrbetrieb, zu erfassen, zu clustern und in einer Wissensbasis zu hinterlegen. Bei vorhandener Wissensbasis kann dann ständig die aktuel- 
le Situation in Bezug auf den vermeintlichen Ausgang der Entwicklung bewertet werden. Hierfür existieren zurzeit keine gesicherten Methoden, teilweise nicht einmal Ideen zur Realisierung. Es scheint jedoch ein Weg zu sein, der begangen werden kann.

Die Fortschritte zur Situationsprädiktion lassen sich extrem schwer voraussagen. Signifikant leistungsfähigere Verfahren sind aber vermutlich nur in einem Zeithorizont von zehn Jahren und mehr zu erwarten.

\section{Literatur}

1. Damböck, D.; Bengler, K.; Farid, M.; Tönert, L.: Übernahmezeiten beim hochautomatisierten Fahren. Tagungsband der VDI-Tagung Fahrerassistenz in München, Jahrgang 15, Seite 16ff, 2012

2. Gold, C.; Damböck, D.; Lorenz, L.; Bengler, K.: "Take over!" How long does it take to get the driver back into the loop? In Proceedings of the Human Factors and Ergonomics Society Annual Meeting, Vol. 57, No. 1, pp. 1938-1942, 2013

3. Winner, H.; Hakuli, S.; Wolf, G. (Eds.): Handbuch Fahrerassistenzsysteme: Grundlagen, Komponenten und Systeme für aktive Sicherheit und Komfort, 2. Auflage (2012), Teubner Verlag

4. Nuss, D.; Reuter, S.; Konrad, M.; Munz, M.; Dietmayer, K.: Using grid maps to reduce the number of false positive measurements in advanced driver assistance systems. IEEE Conference on Intelligent Transportation Systems (ITSC), 2012, pp.1509-1514

5. Bouzouraa, M.; Hofmann, U.: Fusion of occupancy grid mapping and model based object tracking for driver assistance systems using laser and radar sensors. IEEE Intelligent Vehicles Symposium (IV), 2010, pp. 294-300

6. Coué, C.; Pradalier, C.; Laugier, C.; Fraichard, T.; Bessiere, P.: Bayesian Occupancy Filtering for Multitarget Tracking: an Automotive Application. International Journal of Robotics Research, 2006, Vol. 25.1, pp.19-30

7. Konrad, M.; Szczot, M.; Schüle, F.; Dietmayer, K.: Generic grid mapping for road course estimation. IEEE Intelligent Vehicles Symposium (IV), 2011, pp. 851-856

8. Schmid, M.R.; Mählisch, M.; Dickmann, J.; Wünsche, H.-J.: Dynamic level of detail 3d occupancy grids for automotive use. IEEE Intelligent Vehicles Symposium (IV), 2010, pp. 269-274

9. Kalman, R.: A new approach to linear filtering and prediction problems. Transactions of the ASME - Journal of Basic Engineering, 1960, Vol. 82, pp. 35-45

10. Mahler, R.: Statistical Multisource-Multitarget Information Fusion. Artech House, Boston, 2007

11. Bar-Shalom, Y.; Tse, E.: Tracking in a cluttered environment with probabilistic data association. Automatica, 1975, Vol. 11, pp. 451-460

12. Bar-Shalom, Y.; Fortmann, T.: Tracking and Data Association. Academic Press, Boston, 1988

13. Musicki, D.; Evans, R.: Joint Integrated Probabilistic Data Association: JIPDA, IEEE Transactions on Aerospace and Electronic Systems. 2004, Vol. 40.3, pp. 1093-1099

14. Munz, M.: Generisches Sensorfusionsframework zur gleichzeitigen Zustands- und Existenzschätzung für die Fahrzeugumfelderfassung. PhD Thesis, Ulm University, 2011

15. Nuss, D.; Stuebler, M.; Dietmayer, K.: Consistent Environmental Modeling by Use of Occupancy Grid Maps, Digital Road Maps, and Multi-Object Tracking. IEEE Intelligent Vehicles Symposium (IV), 2014

16. Shafer, G.: A Mathematical Theory of Evidence. Princeton University Press, 1976

17. Reuter, S.; Wilking, B.; Wiest, J.; Munz, M.; Dietmayer, K.: Real-time multi-object tracking using random finite sets. IEEE Transactions on Aerospace and Electronic Systems, 2013, Vol. 49.4, pp. 2666-2678 
18. Vo, B.-T.; Vo, B.-N.: Labeled Random Finite Sets and Multi-Object Conjugate Priors. IEEE Transactions on Signal Processing, 2013, Vol. 61, 3460-3475

19. Reuter, S.; Vo, B. T.; Vo, B. N.; Dietmayer, K.: The labeled multi-Bernoulli filter. Signal Processing, IEEE Transactions on, 62(12), pp. 3246-3260, 2014

20. Viola, P.; Jones, M.: Rapid object detection using a boosted cascade of simple features. In Computer Vision and Pattern Recognition, 2001. CVPR 2001. Proceedings of the 2001 IEEE Computer Society Conference on (Vol. 1, pp. I-511). IEEE

21. Egmont-Petersen, M.; de Ridder, D.; Handels, H.: Image processing with neural networks a review. Pattern recognition, 35(10), pp. 2279-2301

22. Zhang, G. P. : Neural networks for classification: a survey. Systems, Man, and Cybernetics, Part C: Applications and Reviews, IEEE Transactions on, 30(4), pp. 451-462

23. Godthelp, H.; Milgram, P.; Blaauw, G. J.: The development of a time-related measure to describe driving strategy. Human Factors: The Journal of the Human Factors and Ergonomics Society, 26(3), 1984, pp.257-268 